\title{
Two-Component Relativistic Hamiltonians and the Douglas-Kroll Approximation
}

\author{
M. BARYSZ \\ Department of Quantum Chemistry, Institute of Chemistry \\ Nicolaus Copernicus University, 7, Gagarin St., 87-100 Toruń, Poland
}

(Received November 15, 2001; in final form March 28, 2002)

\begin{abstract}
The iterative solutions of the previously derived operator equation which defines an open-ended formalism for the reduction of the 4-component Dirac Hamiltonian to 2-component "electronic" operators of arbitrarily high accuracy, are discussed. It is shown that by departing from the approach based solely on the operator algebra one can define the initial iterative solution which leads to the 2-component Douglas-Kroll Hamiltonian. The present derivation reveals the origin of the success of methods based on the Douglas-Kroll Hamiltonian. It also shows that among relatively simple 2-component Hamiltonians, which are exact through the fourth power of the fine structure constant, the Douglas-Kroll operator is the most complete one. Also a computationally convenient and highly compact formula for matrix elements of the Douglas-Kroll Hamiltonian is obtained as a by-product of this investigation.
\end{abstract}

PACS numbers: 31.15.-p, 31.30.Jv

\section{Introduction}

The attempts to reduce the 4-component Dirac Hamiltonian [1] to two mutually independent 2-component operators go back to the early days of quantum mechanics. The formal solution of this problem has been given by Foldy and Wouthuysen (FW) [2] and found to lead to essentially singular operators, making the resulting two-component Hamiltonians useless in applications going beyond the order of $\alpha^{2}$ ( $\alpha=1 / c$, where $c$ is the velocity of light, $c \approx 137.036$ a.u. of velocity). This problem appears to have been resolved in the method proposed 
by Douglas and Kroll (DK) [3]. Owing to its implementation by Hess et al. [4-6] the 2-component DK Hamiltonian has been made into one of the most successful tools of the relativistic quantum chemistry. A competitive 2-component technique, which explicitly avoids the presence of singular operators, has been proposed some years ago by Snijders et al. [7-9].

Recently, Barysz, Sadlej, and Snijders (BSS) [10] have investigated an alternative approach to the block-diagonalization problem. This approach has been formulated in terms of the operator equation, whose solutions determine the unitary transformation to be performed on the Dirac Hamiltonian. Solutions of this equation can be generated iteratively and lead to a family of 2-component Hamiltonians which can be made exact through any desired order in $\alpha^{2}$. The resulting 2-component BSS Hamiltonians which are exact through the given $k$-th order in $\alpha^{2}$ differ by some contaminants of all higher orders in $\alpha^{2}$. The performance of the given $\alpha^{2 k}$-order BSS Hamiltonian $h_{2 k}$ significantly depends on the included portion of these higher order terms [11]. Some general aspects of the relation between the DK and BSS Hamiltonians have been analysed earlier [10]. It has been shown that through terms of the order of $\alpha^{4}$ the $h_{4}$ BSS Hamiltonian is fully equivalent to that of the DK method. This finding has been used to prove that in both cases the one-electron energy eigenvalues are exact through the order of $\alpha^{4}[10]$.

The iterative solution of the operator equation underlying the generation of the 2-component Hamiltonians of arbitrarily high accuracy with respect to $\alpha^{2}$ is a very attractive analytic tool which can be used to gain a deeper insight into the structure and contents of different methods. The purpose of this paper is to further exploit this tool in the investigation of relations between $h_{4}$ and DK Hamiltonians. It will be shown that with a certain particular choice of the solution of the iterative scheme leading to BSS Hamiltonians one obtains the two-component DK Hamiltonian. This analysis reveals the secret of the exceptionally good performance of the DK method and shows how several higher order terms are summed up to infinity. In terms of the present analysis the DK method reappears as possibly the most powerful scheme of the leading order of $\alpha^{4}$ [10].

A brief summary of the BSS approach will be presented in Sec. 2. The iterative solutions of the operator equation for the determination of the block-diagonalizing unitary transformation will be discussed in Sec. 3. It will be shown that one of these solutions leads to a compact two-component Hamiltonian which is fully equivalent to the usual DK operator [3,5]. The proof of this equivalence is given in Appendix. The extension of the $\alpha^{4}$-order theory to higher orders in $\alpha^{2}$ and related issues will be discussed in Sec. 4 .

\section{A survey of the derivation of the BSS Hamiltonians}

The purpose of this survey is mainly to establish the notation used in the present paper. A more detailed derivation of different equations and their dis- 
cussion can be found in Ref. [10]. Let $H_{0}$ be the Dirac-type Hamiltonian for an electron moving in the external potential $V$. In the usual notation $[1,10,12]$

$$
H_{0}=c \boldsymbol{\alpha} p+\beta c^{2}+\left(V-c^{2}\right) I=\left(\begin{array}{cc}
V & c \boldsymbol{\sigma} p \\
c \boldsymbol{\sigma} p & V-2 c^{2}
\end{array}\right) .
$$

The atomic units are used throughout this paper and $c$ denotes the velocity of light. Its inverse $\alpha$ is the usual ordering parameter in the perturbation treatment of relativistic effects $[1,2,13,14]$. Alternatively, one can use the expansion in terms of the coupling between the electron and external fields [3].

The initial step in the development of possibly non-singular two-component theories is the transformation of $H_{0}$ by the exact free-particle Foldy-Wouthuysen (fpFW) unitary transformation $U_{0}[2,3,10]$. The resulting transformed Hamiltonian $H_{1}=U_{0}^{\dagger} H_{0} U_{0}$

$$
H_{1}=\left(\begin{array}{cc}
T_{p}+A\left(V+\alpha^{2} B V B\right) A & \alpha A[V, B] A \\
\alpha A[B, V] A & -2 \alpha^{-2}-T_{p}+A\left(V+\alpha^{2} B V B\right) A
\end{array}\right),
$$

where

$$
A=\sqrt{\frac{e_{p}+1}{2 e_{p}}}, \quad B=\frac{1}{e_{p}+1} \sigma p, \quad e_{p}=\sqrt{1+\alpha^{2} p^{2}},
$$

and

$$
T_{p}=\alpha^{-2}\left(e_{p}-1\right)
$$

is fully equivalent to $H_{0}$. The preliminary fpF W transformation of $H_{0}$ is believed to be responsible for the absence of operators which would lead to severe divergencies in numerical applications [15]. This, however, is concluded mostly on the basis of numerical calculations (see also Ref. [16]).

The next step is to determine a unitary transformation $U$ which brings $H_{0}$ into block-diagonal form

$$
H=U^{\dagger} H_{1} U=\left(\begin{array}{cc}
h_{+} & 0 \\
0 & h_{-}
\end{array}\right) .
$$

This transformation can be chosen in the following form $[10,17]$ :

$$
U=\left(\begin{array}{cc}
\Omega_{+} & R_{-} \Omega_{-} \\
R_{+} \Omega_{+} & \Omega_{-}
\end{array}\right)
$$

where

$$
\Omega_{+}=\left(1+R_{+}^{\dagger} R_{+}\right)^{-1 / 2}, \Omega_{-}=-\left(1+R_{-}^{\dagger} R_{-}\right)^{-1 / 2}, R_{-}=-R_{+}^{\dagger},
$$

and $R_{+}$is the root of the following operator equation [17]:

$$
R=\left[\left(H_{1}\right)_{22}\right]^{-1}\left[-\left(H_{1}\right)_{21}+R\left(H_{1}\right)_{11}+R\left(H_{1}\right)_{12} R\right],
$$

which corresponds to the assumption that $R_{+}$is a "small" operator as compared to the other operators in the r.h.s. of Eq. (8). The symbols $\left(H_{1}\right)_{i j}$ refer to the $(i, j)$-th $2 \times 2$ block of the Hamiltonian $H_{1}$ of Eq. (2). 
Once Eq. (8) is solved, the "electronic" 2-component Hamiltonian $h_{+}$assumes the following form:

$$
\begin{aligned}
h_{+}= & \Omega_{+}^{\dagger}\left(H_{1}\right)_{11} \Omega_{+}+\Omega_{+}^{\dagger} R_{+}^{\dagger}\left(H_{1}\right)_{21} \Omega_{+}+\Omega_{+}^{\dagger}\left(H_{1}\right)_{12} R_{+} \Omega_{+} \\
& +\Omega_{+}^{\dagger} R_{+}^{\dagger}\left(H_{1}\right)_{22} R_{+} \Omega_{+} .
\end{aligned}
$$

Upon combining this equation with iterative solutions of Eq. (8) one can derive a series of approximations $h_{2 k}$ to $h_{+}$, which are exact through the $k$-th order in $\alpha^{2}$ [10]. In this paper particular attention will be given to $h_{4}$ Hamiltonians, i.e., to those which are exact through the order of $\alpha^{4}$.

For the purpose of the present analysis let us consider the explicit form of the operator equation (8). After substituting the appropriate $2 \times 2$ blocks of $H_{1}$ this equation can be written as:

$$
\begin{aligned}
2 R= & \alpha^{3} A[B, V] A-\alpha^{2}\left\{R, T_{p}\right\}_{+}+\alpha^{2}[A V A, R]+\alpha^{4}[A B V B A, R] \\
& -\alpha^{3} R A[V, B] A R,
\end{aligned}
$$

where $\{\ldots, \ldots\}_{+}$denotes the anticommutator. The analysis of the leading order [10] in $\alpha$ for the operators entering Eq. (10) shows that $R$ has the leading order of $\alpha^{3}$. Through this leading order in $\alpha$ the approximate solution for $R$ reads

$$
R \approx \frac{1}{2} \alpha^{3} A[B, V] A
$$

and gives the simplest $h_{4}$ BSS Hamiltonian investigated in Ref. [10]

$$
h_{4}=h_{4}^{(0)}=T_{p}+A\left(V+\alpha^{2} B V B\right) A+\frac{1}{2} \alpha^{4} A[V, B] A A[B, V] A .
$$

This Hamiltonian has been shown to be equivalent to the DK Hamiltonian through terms of the order of $\alpha^{4}$.

\section{Iterative solutions for $R$ and the DK Hamiltonian}

The use of the explicit operator equation (8) without reference to its representation in a certain selected basis set offers quite obvious formal advantages. This technique can be used to derive a variety of 2-component methods which are exact through arbitrarily high order in $\alpha^{2}[10,11]$. However, the use of iterative methods brings about the question of their convergence and the related question of the choice of the initial approximation to $R$. Given some initial approximation $R^{(0)}$ to $R$, the iterative scheme $R^{(n+1)}=f\left(R^{(n)}\right)$ may not necessarily collect the higher-order contributions efficiently enough.

The choice of $R^{(0)}$ in the form given by Eq. (11) was based on the selection of leading contributions with respect to $\alpha$. However, one should note that the anticommutator expression in the r.h.s. of Eq. (10) can be written in the following form:

$$
\alpha^{2}\left\{R, T_{p}\right\}_{+}=\left\{R, e_{p}-1\right\}_{+} .
$$


Thus, the anticommutator becomes apparently of the same order as $R$, though one should remember that the operator $\left(e_{p}-1\right)$ is itself of the leading order of $\alpha^{2}$. The identity (13) immediately suggests that a useful initial approximation, say $\tilde{R}$, to $R$ will be generated by the solution of the following equation:

$$
\left\{\tilde{R}, e_{p}\right\}_{+}=\alpha^{3} A[B, V] A,
$$

which can be cast into manifestly iterative form

$$
\left(1+e_{p}\right) \tilde{R}=\alpha^{3} A[B, V] A-\tilde{R}\left(e_{p}-1\right),
$$

with the solution

$$
\tilde{R}=\sum_{n=0}^{\infty} \frac{(-1)^{n}}{\left(1+\epsilon_{p}\right)^{n+1}} A[B, V] A\left(e_{p}-1\right)^{n} .
$$

One should note that Eq. (14) is equivalent to the commutator equation used in the derivation of the DK Hamiltonian $[3,5]$. In the BSS approach this equation appears as a generator of the initial $R$ which is then to be used in the iterative solution of Eq. (10). To further analyse its solution and to a void the use of infinite summations it is convenient to introduce the matrix representation of the operator $\tilde{R}$.

The basis set representation of solutions of the commutator equations is the common practice in the derivation of the DK Hamiltonian [3, 5]. The solution is obtained by using a basis set which diagonalizes the $p^{2}$ operator*

$$
\left\langle k\left|p^{2}\right| k^{\prime}\right\rangle=w_{k} \delta_{k, k^{\prime}} .
$$

As a consequence the same basis set diagonalizes the $e_{p}, T_{p}, A$, and $B$ operators. In particular

$$
\left\langle k\left|e_{p}\right| k^{\prime}\right\rangle=E_{k} \delta_{k, k^{\prime}} .
$$

Thus, the solution $\tilde{R}$ of Eq. (14) in the basis set $|k\rangle$ becomes

$$
\tilde{R}_{k k^{\prime}}=\left\langle k|\tilde{R}| k^{\prime}\right\rangle=\alpha^{3} \frac{D_{k k^{\prime}}^{\dagger}}{E_{k}+E_{k^{\prime}}},
$$

where

$$
D_{k k^{\prime}}^{\dagger}=\left\langle k\left|D^{\dagger}\right| k^{\prime}\right\rangle=\left\langle k|A[B, V] A| k^{\prime}\right\rangle .
$$

One should note that $\tilde{R}$ is equivalent to the exact $R$ through the order of $\alpha^{3}$ and contains some contributions of all higher orders in $\alpha$. In consequence, the corresponding two-component Hamiltonian $\tilde{h}_{4}$ will be exact through the fourth order in $\alpha$ and can be formally written as

$$
\tilde{h}_{4}=T_{p}+A\left(V+\alpha^{2} B V B\right) A+\frac{1}{2} \alpha\left(A[V, B] A \tilde{R}+\tilde{R}^{\dagger} A[B, V] A\right) .
$$

*In the original derivation of the DK Hamiltonian [3] the set $|k\rangle$ is meant to be the momentum eigenfunctions $|k\rangle$ and the commutator equations are solved for Fourier kernels of the corresponding operators. In implementations of the DK method the momentum basis set is replaced by a set of functions defined in the coordinate space with the usual boundary conditions in infinity [18-20]. The accuracy of this approximation has been recently extensively tested [21]. 
Its matrix elements in the $|k\rangle$ basis set become

$$
\begin{aligned}
& \left\langle k\left|\tilde{h}_{4}\right| k^{\prime}\right\rangle=\left\langle k\left|T_{p}+A\left(V+\alpha^{2} B V B\right) A\right| k^{\prime}\right\rangle \\
& \quad+\frac{1}{2} \alpha^{4} \sum_{k^{\prime \prime}}\left(D_{k k^{\prime \prime}} \frac{1}{E_{k^{\prime \prime}}+E_{k^{\prime}}} D_{k^{\prime \prime} k^{\prime}}^{\dagger}+D_{k k^{\prime \prime}} \frac{1}{E_{k^{\prime \prime}}+E_{k}} D_{k^{\prime \prime} k^{\prime}}^{\dagger}\right) .
\end{aligned}
$$

It can be shown (see Appendix) that the $\alpha^{4}$-order operator given by Eq. (22) is exactly equivalent to the DK Hamiltonian, i.e., $\tilde{h}_{4}=h^{\mathrm{DK}}$ and $\tilde{R}=R^{\mathrm{DK}}$. The form of the DK Hamiltonian derived in this paper is much simpler than that of the original $h^{\mathrm{DK}}[3,5]$. Moreover, the present method of its derivation reveals several aspects of the iterative procedure $[10,11]$ of solving Eq. (10).

\section{Discussion}

The derivation of the DK Hamiltonian presented in this paper shows that it belongs to the class of the $h_{4}$ BSS operators and corresponds to a certain particular form of the initialization of the iterative solution of Eq. (10). It is obvious that in the algebraic approach $[10,17]$ to the block-diagonalization of $H_{1}$ there are infinitely many ways of defining the initial iterative step. The simplest one, given by Eq. (11), was formally analysed in Ref. [10]. Though convenient from the point of view of the formal analysis of the contents of the $h_{2 k}$ Hamiltonians, this approximation is by no means the most efficient one in computational applications. On the other hand, the solution for $R^{\mathrm{DK}}$ is either given by the infinite operator series (16) or in terms of its matrix elements (19).

For implementations of the DK method the use of the highly compact Eq. (22) is obviously advantageous. However, the Hamiltonian defined in terms of its matrix elements becomes basis set dependent. Thus, for the discussion of analytic features of different 2-component Hamiltonians the iterative solutions for $R$ are of certain preference. It is worthwhile to note that by manipulating Eq. (10) several other iterative schemes with explicit solutions for $R$ can be developed [11]. This resembles the strategies used in many-body theories of the electron correlation. Their success depends on the efficiency of partial infinite summations of certain classes of diagrams or in other words, on the choice of the iterative scheme for the solution of certain nonlinear equations [22].

One of the ways of defining the so-called DK2 approximation [3, 5] is by requesting that the corresponding Hamiltonian contains all terms through the second order in the interaction potential $V$. Some preference of this choice has been extensively discussed by Kutzelnigg [15]. According to the derivation presented in this paper the DK2 Hamiltonian can be alternatively classified as a member of the $h_{4}$ class of the BSS Hamiltonians. The difference between various ways of initializing the solution of Eq. (10) amounts to different strategies of including in the l.h.s of this equation as many as possible terms of the higher order in $\alpha^{2}$. In consequence, the leading contribution to the initial $R$ remains of the order $\alpha^{3}$ whereas the (incomplete) contribution of terms of the higher order in $\alpha^{2}$ is hidden among other operators which define the initial solution. This corresponds to partial 
summation of infinite series in $\alpha^{2}$ which is a well-established method of avoiding divergencies in quantum electrodynamics. One may expect that the most successful method derived from the iterative approach to the solution of Eq. (10) should majorize the contribution of these partial sums [11]. According to the present analysis the initial $\tilde{R}$ given by the solution of Eq. (14) is likely to be superior to solutions obtained from other partitions of Eq. (10). This also shows a close resemblance between the methods used to efficiently sum up the series in $\alpha^{2}$ and those employed in the context of many-body theories of the electron correlation.

The derivation of $R^{\mathrm{DK}}$ clearly shows why the DK Hmiltonian should be superior to other Hamiltonians of the BSS $h_{4}$ class. By combining in Eq. (10) the $2 R$ term with the $\alpha^{2}\left\{R, T_{p}\right\}_{+}$term from the r.h.s. of this equation one enhances the implicit summation of the higher order contributions. This can be seen from the resulting operator equation (14), where $e_{p}$ has the leading term of the zeroth order in $\alpha^{2}$ [10]. Although the efficiency of this rearrangement of Eq. (10) should be higher than that of other iterative approaches, the convenience of using the convenient formalism of the operator algebra is lost.

The development of 2-component Hamiltonians $h_{2 k}, k \geq 3$ can be accomplished either in terms of purely algebraic iterative solutions of the operator equation (10) or by iterating its matrix counterpart. For sufficiently high values of $k$ the two methods, which differ in higher order contributions, should give $h_{2 k}, k \geq 3$ Hamiltonians of essentially the same accuracy. With the given initial approximation to the solution of Eq. (10), the iterative solutions can be ordered either with respect to the powers of $\alpha$ or with respect to the powers of the coupling parameter $V$.

The first choice was advocated in Ref. [10]. The formulation of the block-diagonalization problem presented in this paper gives a possibility of selecting the higher order $h_{2 k}$ Hamiltonians $[10,11]$ by their ordering with respect to the leading powers [10] of $\alpha^{2}$. The use of the coupling parameter is more in the spirit of the original formulation of the DK method [3-5]. By the well-known rules of the perturbation calculus, the contribution of the third order in $V$ must be totally expressible [23] in terms of the transformation which gives the DK2 Hamiltonian. This and higher order contributions are implicit in earlier derivations of the DK Hamiltonian $[4,5,10]$. The $V^{3}$-order terms have been recently discussed by Nakajima and Hirao [24]. It can be shown [10] that the DK Hamiltonian augmented by these terms (DK3 Hamiltonian of Nakajima and Hirao [24]) is a member of the $h_{6}$ class of the BSS Hamiltonians.

\section{Acknowledgments}

The author acknowledges the financial support from the State Committee for Scientific Research (Contract No. 3 T09A 116 17). The reported research is also a part of activities within the COST-D9 WG0009 "PAMALOF" action. 


\section{Appendix}

To show that the DK Hamiltonian derived in this paper is equivalent to the one presented by other authors $[3,5]$ one needs to discuss only the term of the $\alpha^{4}$ order, i.e., the sum contributing to the r.h.s. of Eq. (22). In the usual $[4,5]$ form of the DK Hamiltonian the relevant term is written as

$$
h 4=-\frac{1}{2} \alpha^{-2}\left[W_{1},\left[W_{1}, \beta e_{p}\right]\right],
$$

where the operator $W_{1}$ is defined in terms of its matrix elements

$$
\left\langle k\left|W_{1}\right| k^{\prime}\right\rangle=\alpha^{3} \beta \frac{\left(O_{1}\right)_{k k^{\prime}}}{E_{k}+E_{k^{\prime}}}
$$

and

$$
O_{1}=\beta A[\mathcal{R}, V] A=-O_{1}^{\dagger}
$$

with

$$
\mathcal{R}=\frac{\boldsymbol{\alpha} \boldsymbol{p}}{1+e_{p}} .
$$

Note that the units are adapted to our definitions of operators.

Taking into account that [1]

$$
\boldsymbol{\alpha} \beta=-\beta \boldsymbol{\alpha} \text { and } \beta^{2}=1,
$$

the matrix element $\left\langle k|h 4| k^{\prime}\right\rangle=(h 4)_{k k^{\prime}}$ of (23) becomes

$$
\begin{gathered}
(h 4)_{k k^{\prime}}=-\frac{1}{2} \alpha^{4} \beta \sum_{k^{\prime \prime}} \frac{\left(O_{1}\right)_{k k^{\prime \prime}}\left(O_{1}\right)_{k^{\prime \prime} k^{\prime}}}{\left(E_{k}+E_{k^{\prime \prime}}\right)\left(E_{k^{\prime \prime}}+E_{k^{\prime}}\right)}\left(E_{k^{\prime}}+E_{k}+2 E_{k^{\prime \prime}}\right) \\
=\frac{1}{2} \alpha^{4} \beta \sum_{k^{\prime \prime}}\left(\left(O_{1}\right)_{k k^{\prime \prime}} \frac{1}{E_{k}+E_{k^{\prime \prime}}}\left(O_{1}^{\dagger}\right)_{k^{\prime \prime} k^{\prime}}+\left(O_{1}\right)_{k k^{\prime \prime}} \frac{1}{E_{k^{\prime \prime}}+E_{k^{\prime}}}\left(O_{1}^{\dagger}\right)_{k^{\prime \prime} k^{\prime}}\right) .
\end{gathered}
$$

The $(1,1)$ block of this $4 \times 4$ matrix is exactly equivalent to the matrix element (22) of the 2-component DK Hamiltonian.

\section{References}

[1] R.E. Moss, Advanced Molecular Quantum Mechanics, Chapman and Hall, London 1973.

[2] L.L. Foldy, S.A. Wouthuysen, Phys. Rev. 78, 29 (1950).

[3] M. Douglas, N.M. Kroll, Ann. Phys. 82, 89 (1974).

[4] B.A. Hess, Phys. Rev. A 33, 3742 (1986).

[5] G. Jansen, B.A. Hess, Phys. Rev A 39, 6016 (1989).

[6] R. Samzow, B.A. Hess, G. Jansen, J. Chem. Phys. 96, 1227 (1992).

[7] E. van Lenthe, E.J. Baerends, J.G. Snijders, J. Chem. Phys. 99, 4597 (1993). 
[8] A.J. Sadlej, J.G. Snijders, E. van Lenthe, E.J. Baerends, J. Chem. Phys. 102, 1758 (1995).

[9] E. van Lenthe, R. van Leeuwen, E.J. Baerends, J.G. Snijders, Int. J. Quantum Chem. 57, 281 (1996).

[10] M. Barysz, A.J. Sadlej, J.G. Snijders, Int. J. Quantum Chem. 65, 225 (1997).

[11] M. Barysz, J. Chem. Phys. 114, 9315 (2001).

[12] V. Kellö, A.J. Sadlej, Int. J. Quantum. Chem. 68, 159 (1998).

[13] W. Kutzelnigg, Z. Phys. D 11, 15 (1989).

[14] W. Kutzelnigg, Z. Phys. D 15, 27 (1990).

[15] W. Kutzelnigg, Chem. Phys. 225, 203 (1990).

[16] G. Hardekopf, J. Sucher, Phys. Rev. A 30, 703 (1984).

[17] J.-L. Heully, I. Lindgren, E. Lindroth, S. Lundqvist, A.J.-M. Mårtensson-Pendril, J. Phys. B 19, 2799 (1986).

[18] B.A. Hess, Phys. Rev. A 22, 756 (1985).

[19] J. Almlöf, K. Feegri, Jr., H.H. Grelland, Chem. Phys. Lett. 114, 53 (1985).

[20] B.A. Hess, R.J. Buenker, P. Chandra, Int. J. Quantum Chem. 29, 737 (1986).

[21] D. Woźniak, A.J. Sadlej, Acta Phys. Pol. A 98, 673 (2000).

[22] R.J. Bartlett, in: Methods in Computational Chemistry, Advanced Series in Physical Chemistry, Ed. D.R. Yarkony, Vol. 2, World Scientific, Singapore 1995, p. 1047 and references therein.

[23] J.D. Bjorken, S.D. Drell, Relativistic Quantum Fields, McGraw-Hill, New York 1965.

[24] T. Nakajima, K. Hirao, J. Chem. Phys. 113, 7786 (2000). 